\title{
Synthesis of thieno[2,3-d]thiazole-6-carboxylic acid derivatives as potential inducers of systemic resistance in plants
}

\author{
Peter Stanetty*, Thomas Dvorak, and Marko D. Mihovilovic \\ Institute of Organic Chemistry, Vienna University of Technology Getreidemarkt 9/154, A-1060 \\ Vienna, Austria. Fax: +43-1-58801-15494 \\ E-mail:pstanett@pop.tuwien.ac.at
}

Dedicated to Prof. Fritz Sauter on the occasion of his $70^{\text {th }}$ birthday
(received 20 Nov 00; accepted 28 Oct 01; published on the web 05 Nov 01)

\begin{abstract}
Reduction of nitrothiocyanatothiophene 2, available via nucleophilic substitution from the corresponding bromo compound $\mathbf{1}$, gave thienothiazole $\mathbf{3}$ in excellent yield. Conversion of the amino functionality under Sandmeyer conditions gave access to halo compounds $\mathbf{4}$ as key intermediates for the subsequent introduction of sulfur, nitrogen, and oxygen nucleophiles. An unexpected side-reaction was observed when DMF was used as solvent and a mechanism for the introduction of a dimethylamino group is proposed.
\end{abstract}

Keywords: Thiazoles, thienothiazoles, plant activators, nucleophilic substitution

\section{Introduction}

Treatment of crop plants with plant activators - a recently developed group of synthetic compounds - triggers their own defense system giving them the ability to resist the attack of naturally occurring pathogens ("systemic acquired resistance", SAR). ${ }^{1}$ Recently, acibenzolar-Smethyl $\left(\right.$ Bion $\left.^{\circledR}, \mathbf{I}\right)$ was introduced to the market as the first commercial product in this field of research. After succeeding in the synthesis of similar thienothiadiazole derivatives (II) ${ }^{2}$ we focused on various derivatives of thieno[2,3-d]thiazoles III.<smiles>CC(=O)c1cccc2nnsc12</smiles>

I Acibenzolar-S-methyl; Bion ${ }^{\circledR}$

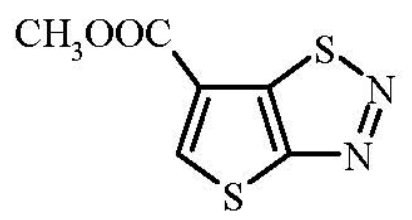

II

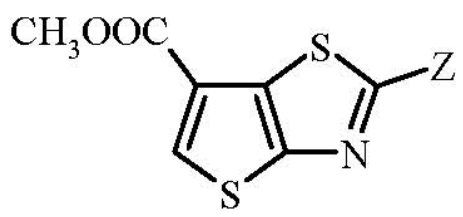

III $Z=$ Hal, OR, SR, NRR' 


\section{Results and Discussion}

Starting from 1, available via a recently optimized protocol, ${ }^{3}$ nucleophilic displacement of the bromo substituent by sodium thiocyanate led to intermediate 2 in excellent yield (Scheme 1). The nitro group in $\mathbf{2}$ was reduced with $\mathrm{Fe} / \mathrm{AcOH}$ to the corresponding thiophenamine $\mathbf{2}$ which spontaneously cyclized via intramolecular nucleophilic attack at the carbon of the SCN-group yielding the amino compound $\mathbf{3}$.

Our goal was the development of a highly flexible route to compounds III with a variety of substituents $Z$. Hence the corresponding halo products $(Z=H a l$, Scheme 1$)$ represent valuable intermediates. Introduction of chlorine and formation of the chloro derivative $\mathbf{4}$ was achieved by diazotization under non-aqueous conditions followed by treatment of the diazonium salt with $\mathrm{CuCl}_{2}{ }^{4}$ However, the reaction of 3 with $\mathrm{CuBr}_{2}$ under comparable conditions gave a mixture of the desired $5 \mathbf{a}$ accompanied by substantial amounts of the dibromo compound $\mathbf{5 b}$ as a byproduct.

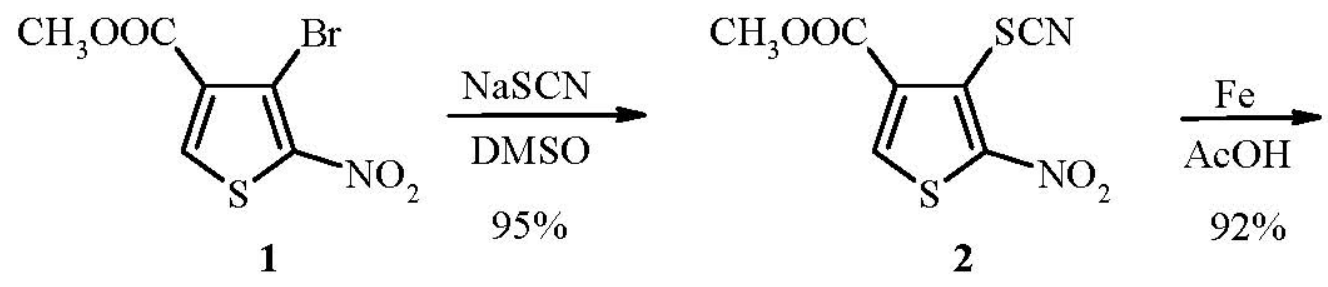

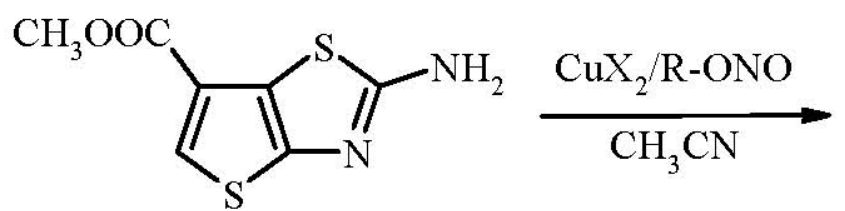

3

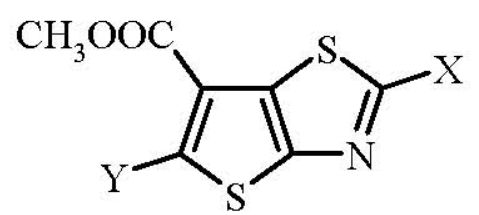

4: $\mathrm{X}=\mathrm{Cl}, \mathrm{Y}=\mathrm{H}, 50 \%$

5a: $\mathrm{X}=\mathrm{Br}, \mathrm{Y}=\mathrm{H}, 26 \%$

5b: $\mathrm{X}=\mathrm{Br}, \mathrm{Y}=\mathrm{Br}, 12 \%$

\section{Scheme 1}

A series of 2-substituted thienothiazoles 6-15 was available via nucleophilic substitution of the chloro atom in compound $\mathbf{4}$ applying various sulfur, nitrogen, and oxygen nucleophiles. As expected, sulfur nucleophiles (Scheme 2, entries 1-4) gave excellent conversions to the desired products 6-9. Formation of amines 10-14 (Scheme 2, entries 5-9) required more drastic reaction conditions. A similar behavior was encountered with an oxygen nucleophile (15, Scheme 2, entry $10)$.

A noteworthy side-reaction was observed when using diethanolamine as nucleophile in DMF as solvent. The formation of the expected 12 (yield 34\%) was accompanied by the dimethylamino product $13(15 \%)$. This can be explained by a transamidation reaction of the 
diethanolamine and DMF leading to dimethylamine as a competing nucleophile. A similar reaction was observed by Cho et al. $^{5}$ for the conversion of $\mathbf{V}$ to VII via the following mechanism (Scheme 3).

Essential for the formation of the dimethylamino derivative is the lower reactivity of the amino alcohol towards direct substitution, which can be explained by intramolecular hydrogen bonding. The activated hydroxy group attacks DMF assisted by the adjacent basic amino function to produce an intermediate, which can introduce the dimethylamino group into the substrate while a formate is formed. Subsequent intramolecular transfer of the formyl group occurs under the reaction condition finally to produce formamide VIII.
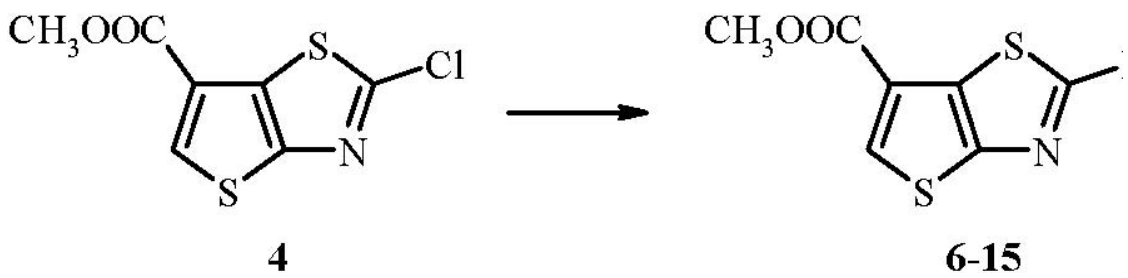

\begin{tabular}{|c|c|c|c|}
\hline Entry & $\mathrm{R}$ & Product & Yield [\%] \\
\hline 1 & $\mathrm{SPh}$ & 6 & 85 \\
\hline 2 & $\mathrm{SCH}_{2} \mathrm{Ph}$ & 7 & 67 \\
\hline 3 & $\mathrm{SCH}_{2} \mathrm{CH}_{2} \mathrm{CH}_{3}$ & 8 & 84 \\
\hline 4 & $\mathrm{SCH}_{2} \mathrm{COOCH}_{3}$ & 9 & 85 \\
\hline 5 & & 10 & 55 \\
\hline 6 & & 11 & 25 \\
\hline 7 & & 12 & 34 \\
\hline 8 & $\mathrm{~N}(\mathrm{Me})_{2}$ & 13 & 15 \\
\hline 9 & $\begin{array}{l}\mathrm{N} \\
\mathrm{H}\end{array}$ & 14 & 47 \\
\hline 10 & OMe & 15 & 40 \\
\hline
\end{tabular}

\section{Scheme 2}




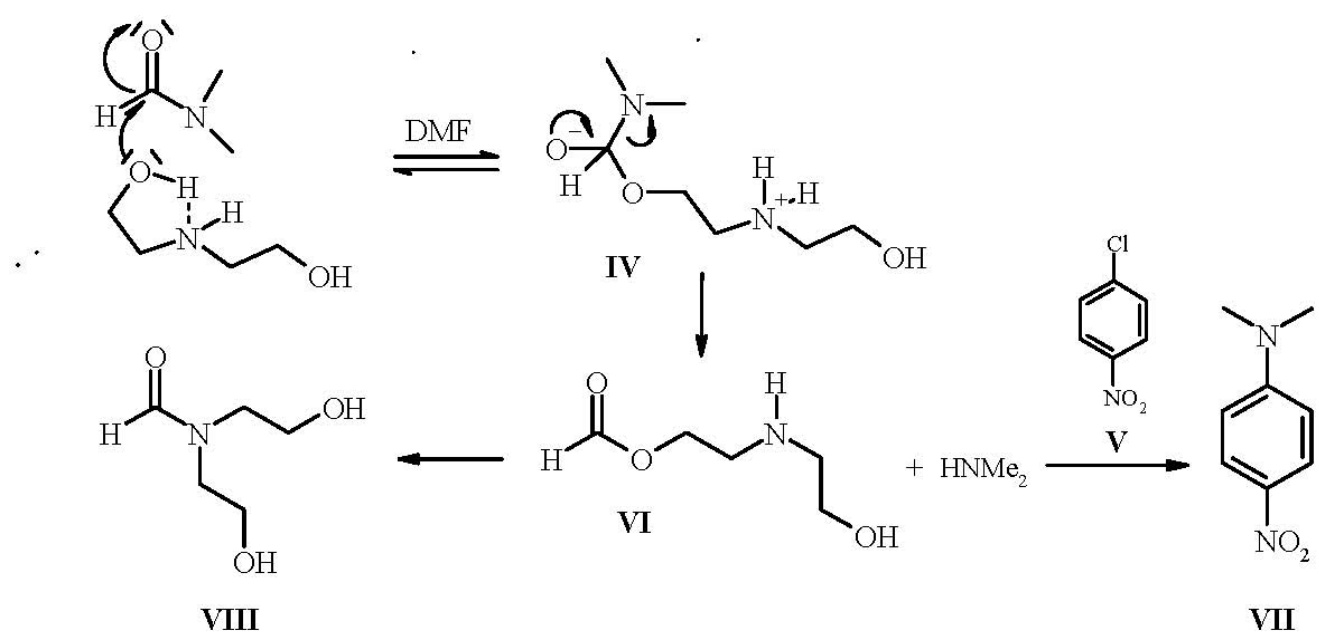

Scheme 3

\section{Experimental Section}

General Procedures. All reactions were carried out with commercially available chemicals, solvents were distilled prior to use. Flash column chromatography (FCC) was performed on Merck Kieselgel $60(40-63 \mu \mathrm{m})$. Melting points were determined on a Galen III Kofler type hotstage apparatus. NMR spectra were recorded with a Bruker AC 200 FT spectrometer $\left({ }^{1} \mathrm{H}\right.$ NMR at $200 \mathrm{MHz})$ with $\mathrm{CDCl}_{3}$ or DMSO- $d_{6}$ as solvents and $\left(\mathrm{CH}_{3}\right)_{4} \mathrm{Si}$ as internal standard. Signals, which could not be assigned unambiguously are marked $(*)$. Elemental analyses were carried out at Microanalytical Laboratory, University of Vienna.

Methyl 5-nitro-4-thiocyanatothiophene-3-carboxylate (2). A solution of methyl 4-bromo-5nitrothiophene-3-carboxylate (1) $(5.00 \mathrm{~g}, 18.79 \mathrm{mmol})$ and dry $\mathrm{NaSCN}$ (4.60 g, $56.38 \mathrm{mmol})$ in dry DMSO $(50 \mathrm{~mL})$ was stirred at $60^{\circ} \mathrm{C}$ for $2 \mathrm{~h}$. The reaction mixture was poured into water, the precipitate formed was filtered off, washed with water, and dried in vacuo to give $\mathbf{1}$ as beige crystals $(4.36 \mathrm{~g}, 95 \%)$; mp $99-100{ }^{\circ} \mathrm{C} ;{ }^{1} \mathrm{H} \mathrm{NMR}\left(\mathrm{CDCl}_{3}\right): \delta 3.99\left(\mathrm{~s}, 3 \mathrm{H}, \mathrm{OCH}_{3}\right), 8.32(\mathrm{~s}, 1 \mathrm{H}, \mathrm{H}-$ 2); ${ }_{13} \mathrm{C} \mathrm{NMR}\left(\mathrm{CDCl}_{3}\right): \delta 53.0\left(\mathrm{q}, \mathrm{OCH}_{3}\right), 107.6(\mathrm{~s}, \mathrm{SCN}), 122.9$ (s, C-4), 133.0 (s, C-3), 136.5 (d, C-2), 150.6 (s, C-5), 160.3 (s, CO . Anal. Calcd. for $\mathrm{C}_{7} \mathrm{H}_{4} \mathrm{~N}_{2} \mathrm{O}_{4} \mathrm{~S}_{2}(244.25)$ : C, 34.42; H, 1.65; N, 11.47. Found: C, 34.58; H, 1.66; N, 11.45 .

Methyl 2-aminothieno[2,3-d]thiazole-6-carboxylate (3). A solution of methyl 5-nitro-4thiocyanatothiophene-3-carboxylate (2) (4.00 g, $16.38 \mathrm{mmol})$ in AcOH (120 mL) was treated with Fe powder $(3.66 \mathrm{~g}, 65.51 \mathrm{mmol})$ in small portions so that the temperature of the reaction mixture was kept below $30{ }^{\circ} \mathrm{C}$. After stirring at room temperature for $1 \mathrm{~h}$ the reaction mixture was poured into a saturated $\mathrm{NaHCO}_{3}$ solution and extracted with diethyl ether. The organic layer was separated and washed with saturated $\mathrm{NaHCO}_{3}$ solution, dried over $\mathrm{Na}_{2} \mathrm{SO}_{4}$ and evaporated to dryness. The crude product was purified by column chromatography (100 g silica gel PE/EtOAc $5: 1)$ to give 3 as orange crystals $(3.22 \mathrm{~g}, 92 \%)$; mp $214-216{ }^{\circ} \mathrm{C} ;{ }^{1} \mathrm{H}$ NMR (DMSO- $\left.d_{6}\right): \delta 3.83(\mathrm{~s}$, $3 \mathrm{H}, \mathrm{OCH}_{3}$ ), 7.49 (broad s, 2H, $\left.\mathrm{NH}_{2}\right), 7.89$ (s, 1H, H-5); ${ }^{13} \mathrm{C}$ NMR (DMSO-d $): \delta 52.0\left(\mathrm{q}, \mathrm{OCH}_{3}\right)$, 
120.9 (s, C-6a*), 123.6 (s, C-6*), 125.5 (d, C-5), 152.6 (s, C-3a), 161.7 (s, CO2), 172.5 (s, C-2).

\section{General procedure for the diazotization of 3}

3-Methylbutylnitrite (1.5 equiv.) was added to a suspension of copper(II) chloride or copper(II) bromide (1.2 equiv.) in dry acetonitrile. The reaction mixture was stirred at room temperature for 10 min, and methyl 2-aminothieno[2,3-d]thiazole-6-carboxylate 3 ( 1 equiv.) was added at $15{ }^{\circ} \mathrm{C}$ within $1 \mathrm{~h}$. The reaction mixture was stirred at room temperature for $30 \mathrm{~min}$ and then filtered. Some silica gel was added, the filtrate was evaporated, and the crude product was purified by column chromatography (silica gel, PE/EtOAc 50:1).

Methyl 2-chlorothieno[2,3-d]thiazole-6-carboxylate (4). Treatment of 3 (0.20 g, $0.94 \mathrm{mmol})$ with $\mathrm{CuCl}_{2}$ gave 4 as yellow crystals $(0.11 \mathrm{~g}, 50 \%)$; mp 155-157 ${ }^{\circ} \mathrm{C} ;{ }^{1} \mathrm{H} \mathrm{NMR}\left(\mathrm{CDCl}_{3}\right): \delta 3.96$ $\left(\mathrm{s}, 3 \mathrm{H}, \mathrm{OCH}_{3}\right), 8.20(\mathrm{~s}, 1 \mathrm{H}, \mathrm{H}-2) ;{ }^{13} \mathrm{C} \mathrm{NMR}\left(\mathrm{CDCl}_{3}\right): \delta 52.3\left(\mathrm{q}, \mathrm{OCH}_{3}\right), 124.0(\mathrm{~s}, \mathrm{C}-6), 132.2(\mathrm{~s}$,

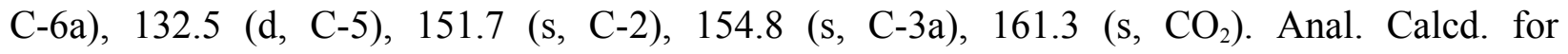
$\mathrm{C}_{7} \mathrm{H}_{4} \mathrm{ClNO}_{2} \mathrm{~S}$ (233.70): C, 35.98; H, 1.73; N, 5.99;. Found: C, 36.18; H, 1.66; N, 5.89.

Methyl 2-bromothieno[2,3-d]thiazole-6-carboxylate (5a). Treatment of $3(0.30 \mathrm{~g}, 1.41 \mathrm{mmol})$ with $\mathrm{CuBr}_{2}$ gave $5 \mathrm{a}$ as colorless crystals $(0.10 \mathrm{~g}, 26 \%)$; mp 161-164 ${ }^{\circ} \mathrm{C} ;{ }^{1} \mathrm{H} \mathrm{NMR}\left(\mathrm{CDCl}_{3}\right): \delta$ $3.95\left(\mathrm{~s}, 3 \mathrm{H}, \mathrm{OCH}_{3}\right), 8.21(\mathrm{~s}, 1 \mathrm{H}, \mathrm{H}-2) ;{ }^{13} \mathrm{C} \mathrm{NMR}\left(\mathrm{CDCl}_{3}\right): \delta 52.4\left(\mathrm{q}, \mathrm{OCH}_{3}\right), 123.8(\mathrm{~s}, \mathrm{C}-6), 132.9$ (d, C-5), 134.0 (s, C-6a), 139.5 (s, C-2), 153.2 (s, C-3a), 161.4 (s, $\mathrm{CO}_{2}$ ). Anal. Calcd. for $\mathrm{C}_{7} \mathrm{H}_{4} \mathrm{BrNO}_{2} \mathrm{~S}_{2}$ (278.15): C, 30.23; H, 1.45; N, 5.04). Found: C, 30.33; H, 1.36; N, 4.86.

Methyl 2,5-dibromothieno[2,3-d]thiazole-6-carboxylate (5b). By-product from the synthesis of compound 5a: Colorless crystals 5b $(0.06 \mathrm{~g}, 12 \%)$; mp 165-167 ${ }^{\circ} \mathrm{C} ;{ }^{1} \mathrm{H} \mathrm{NMR}\left(\mathrm{CDCl}_{3}\right): \delta 3.99$ $\left(\mathrm{s}, 3 \mathrm{H}, \mathrm{OCH}_{3}\right) ;{ }^{13} \mathrm{C} \mathrm{NMR}\left(\mathrm{CDCl}_{3}\right): \delta 52.4\left(\mathrm{q}, \mathrm{OCH}_{3}\right), 121.4(\mathrm{~s}, \mathrm{C}-5), 122.0(\mathrm{~s}, \mathrm{C}-6), 133.7(\mathrm{~s}, \mathrm{C}-$ 6a), 139.7 (s, C-2), 150.7 (s, C-3a), 160.5 (s, $\left.\mathrm{CO}_{2}\right)$.

\section{General procedure for the substitution of $\mathbf{4}$ with sulfur nucleophiles}

The nucleophile (1.1 equiv.) was added to a solution of methyl 2-chlorothieno[2,3-d]thiazole-6carboxylate 4 (1 equiv.) and $\mathrm{K}_{2} \mathrm{CO}_{3}$ (1.1 equiv.) in dry DMF or DMSO. When the reaction was complete the mixture was poured into water. The precipitate formed was filtered off, washed with water, and dried in vacuo. Alternatively, the aqueous solution was repeatedly extracted with diethyl ether, the combined organic layers were washed with saturated aqueous $\mathrm{NaHCO}_{3}$ solution, dried over $\mathrm{Na}_{2} \mathrm{SO}_{4}$, and evaporated to dryness.

Methyl 2-(phenylsulfanyl)thieno[2,3-d]thiazole-6-carboxylate (6). Compound 4 (0.10 g, $0.43 \mathrm{mmol}$ ) reacted with benzenethiol in DMF at room temperature for $1 \mathrm{~h}$ and afforded after purification by column chromatography (silica gel/crude product 100:1, PE/EtOAc 10:1) yellow crystals $6(0.11 \mathrm{~g}, 85 \%)$; mp 93-95 ${ }^{\circ} \mathrm{C} .{ }^{1} \mathrm{H}$ NMR $\left(\mathrm{CDCl}_{3}\right): \delta 3.76\left(\mathrm{~s}, 3 \mathrm{H}, \mathrm{OCH}_{3}\right), 7.25-7.40(\mathrm{~m}$, $3 \mathrm{H}, \mathrm{HPh}-2,4,6)$, 7.47-7.62 (m, 2H, HPh-3,5), 7.94 (s, 1H, H-5); ${ }^{13} \mathrm{C} \mathrm{NMR}\left(\mathrm{CDCl}_{3}\right): \delta 52.2$ (q, $\mathrm{OCH}_{3}$ ), 123.8 (s, C-6), 129.9 (d, CPh-2,6), 130.0 (d, CPh-4), 130.3 (s, CPh-1), 131.7 (d, C-5), 132.1 (s, C-6a), 134.3 (d, Cph-3,5), 155.1 (s, C-3a), 161.6 (s, CO2), 170.8 (s, C-2). Anal. Calcd. for $\mathrm{C}_{13} \mathrm{H}_{9} \mathrm{NO}_{2} \mathrm{~S}_{3}$ (307.42): C, 50.79; H, 2.95; N 4.56. Found: C, 50.87; H, 3.11; N 4.46.

Methyl 2-(benzylsulfanyl)thieno[2,3-d]thiazole-6-carboxylate (7). Compound 4 (1.20 g, 
$5.13 \mathrm{mmol}$ ), nucleophile: benzylthiol, solvent: DMSO, reaction conditions: $60{ }^{\circ} \mathrm{C}, 4 \mathrm{~h}$, purification by column chromatography: (silica gel/crude product 50:1, PE/EtOAc 10:1): light yellow crystals $7(1.10 \mathrm{~g}, 67 \%)$; mp $71-74{ }^{\circ} \mathrm{C}$; ${ }^{1} \mathrm{H}$ NMR $\left(\mathrm{CDCl}_{3}\right): \delta 3.91\left(\mathrm{~s}, 3 \mathrm{H}, \mathrm{OCH}_{3}\right), 4.50(\mathrm{~s}$, $\left.2 \mathrm{H}, \mathrm{SCH}_{2}\right), 7.23-7.44\left(\mathrm{~m}, 5 \mathrm{H}, \mathrm{C}_{6} \mathrm{H}_{5}\right), 8.07(\mathrm{~s}, 1 \mathrm{H}, \mathrm{H}-5) ;{ }^{13} \mathrm{C} \mathrm{NMR}\left(\mathrm{CDCl}_{3}\right): \delta 38.8\left(\mathrm{t}, \mathrm{SCH}_{2}\right), 52.2$ $\left(\mathrm{q}, \mathrm{OCH}_{3}\right), 123.8(\mathrm{~s}, \mathrm{C}-6), 127.7$ (d, CPh-4), $128.6(2 \times \mathrm{d}, \mathrm{CPh}-3,5), 128.9(2 \times \mathrm{d}, \mathrm{CPh}-2,6), 129.1(\mathrm{~s}$, C-6a), 131.3 (d, C-5), 135.8 (s, CPh-1), 154.7 (s, C-3a), 161.6 (s, CO2), 168.8 (s, C-2). Anal. Calcd. for $\mathrm{C}_{14} \mathrm{H}_{11} \mathrm{NO}_{2} \mathrm{~S}_{3}$ (321.44): C, 52.31; H, 3.45; N, 4.36. Found: C, 52.60; H, 3.47; N, 4.09.

Methyl 2-(propylsulfanyl)thieno[2,3-d]thiazole-6-carboxylate (8). Compound 4 (2.00 g, $8.56 \mathrm{mmol}$ ), nucleophile: propane-1-thiol, solvent: DMSO, reaction conditions: $50{ }^{\circ} \mathrm{C}, 4 \mathrm{~h}$, room temperature, overnight, purification by column chromatography (silica gel/product 50:1, PE/EtOAc 10:1): light yellow crystals 8 (1.96 g, 84\%); mp 37-39 ${ }^{\circ} \mathrm{C} .{ }^{1} \mathrm{H} \mathrm{NMR}\left(\mathrm{CDCl}_{3}\right): \delta 1.06$ $\left(\mathrm{t}, J_{3^{\prime}, 2^{\prime}}=6 \mathrm{~Hz}, 3 \mathrm{H}, 3^{\prime}-\mathrm{CH}_{3}\right), 1.83\left(\mathrm{sext}, J_{2^{\prime}, 1^{\prime}}=6 \mathrm{~Hz}, J_{2^{\prime}, 3^{\prime}}=6 \mathrm{~Hz}, 2 \mathrm{H}, 2^{\prime}-\mathrm{CH}_{2}\right), 3.25\left(\mathrm{t}, J_{1^{\prime}, 2^{\prime}}=6 \mathrm{~Hz}\right.$,

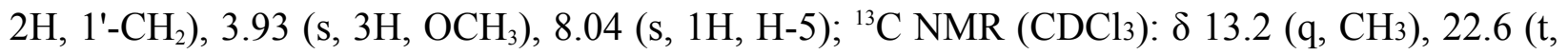
$\mathrm{CH}_{2}$ ), 36.5 (t, SCH 2 ), 52.2 (q, $\mathrm{OCH}_{3}$ ), 123.8 (s, C-6), 130.9 (s, C-6a), 131.1 (d, C-5), 154.8 (s, C3a), 161.7 (s, $\mathrm{CO}_{2}$ ), 170.1 (s, C-2). Anal. Calcd. for $\mathrm{C}_{10} \mathrm{H}_{11} \mathrm{NO}_{2} \mathrm{~S}_{3}(273.40)$ : C, 43.93; H, 4.06; N, 5.12. Found: C, 44.23; H, 3.87; N, 5.10.

Methyl 2-[(methoxy-2-oxoethyl)sulfanyl]thieno[2,3-d]thiazole-6-carboxylate (9). Compound 4 (2.00 g, $8.56 \mathrm{mmol})$, nucleophile: methyl 2-sulfanylacetate, solvent: DMSO, reaction conditions: $70{ }^{\circ} \mathrm{C}, 3 \mathrm{~h}$, purification by column chromatography (silica gel/product 50:1, PE/EtOAc 10:1): colorless crystals 9 (2.22 g, 85\%); mp 80-82 ${ }^{\circ} \mathrm{C} .{ }^{1} \mathrm{H} \mathrm{NMR}\left(\mathrm{CDCl}_{3}\right): \delta 3.74(\mathrm{~s}$, $\left.3 \mathrm{H}, \mathrm{CH}_{2} \mathrm{COOCH}_{3}\right), 3.90\left(\mathrm{~s}, 3 \mathrm{H}, \mathrm{OCH}_{3}\right), 4.07\left(\mathrm{~s}, 2 \mathrm{H}, \mathrm{SCH}_{2}\right), 8.05(\mathrm{~s}, 1 \mathrm{H}, \mathrm{H}-5)$; ${ }^{13} \mathrm{C} \mathrm{NMR}$ $\left(\mathrm{CDCl}_{3}\right): \delta 35.6\left(\mathrm{t}, \mathrm{SCH}_{2}\right), 52.2\left(\mathrm{q}, \mathrm{CH}_{2} \mathrm{CO}_{2} \mathrm{CH}_{3}\right), 52.8\left(\mathrm{q}, \mathrm{OCH}_{3}\right), 123.7(\mathrm{~s}, \mathrm{C}-6 \mathrm{a}), 131.5(\mathrm{~s}, \mathrm{C}-6)$, 131.6 (d, C-5), 154.4 (s, C-3a), 161.5 (s, 6- $\mathrm{CO}_{2}$ ), 167.1 (s, C-2), 168.5 (s, $\left.\mathrm{CH}_{2} \mathrm{COO}\right)$. Anal. Calcd. for $\mathrm{C}_{10} \mathrm{H}_{9} \mathrm{NO}_{4} \mathrm{~S}_{3}$ (303.38): C, 39.59; H, 2.99; N, 4.62. Found: C, 39.39; H, 2.80; N, 4.45.

Methyl 2-pyrrolidinothieno[2,3-d]thiazole-6-carboxylate (10). A solution of $4(0.10 \mathrm{~g}$, $0.43 \mathrm{mmol})$ in dry DMF $(5 \mathrm{~mL})$ was added to a solution of pyrrolidine $(0.06 \mathrm{~g}, 0.43 \mathrm{mmol})$ in DMF $(5 \mathrm{~mL})$. After stirring at room temperature for $2 \mathrm{~h}$ pyrrolidine $(0.06 \mathrm{~g}, 0.43 \mathrm{mmol})$ was added, and the reaction mixture was stirred at room temperature for 1 hour. The solution was then poured into water, and the mixture was extracted with $\mathrm{CH}_{2} \mathrm{Cl}_{2}$. The combined organic layers were washed with $2 \mathrm{~N} \mathrm{HCl}$ and with saturated $\mathrm{NaHCO}_{3}$ solution, dried over $\mathrm{Na}_{2} \mathrm{SO}_{4}$ and evaporated to dryness. The product was purified by column chromatography (silica gel/product 30:1, PE/EtOAc 10:1) to give yellow crystals 10 (0.06 g, 55\%); mp 130-132 ${ }^{\circ} \mathrm{C}$; ${ }^{1} \mathrm{H}$ NMR $\left(\mathrm{CDCl}_{3}\right): \delta 1.95-2.12\left(\mathrm{~m}, 4 \mathrm{H}, 2 \mathrm{CH}_{2}\right), 3.43-3.56\left(\mathrm{~m}, 4 \mathrm{H}, 2 \mathrm{CH}_{2} \mathrm{~N}\right), 3.88\left(\mathrm{~s}, 3 \mathrm{H}, \mathrm{OCH}_{3}\right), 7.62(\mathrm{~s}$, $1 \mathrm{H}, \mathrm{H}-5) ;{ }^{13} \mathrm{C} \mathrm{NMR}\left(\mathrm{CDCl}_{3}\right): \delta 25.5\left(\mathrm{t}, 2 \mathrm{CH}_{2}\right), 49.2\left(\mathrm{t}, 2 \mathrm{CH}_{2} \mathrm{~N}\right), 51.9\left(\mathrm{q}, \mathrm{OCH}_{3}\right), 121.1(\mathrm{~s}, \mathrm{C}-6)$, 124.0 (s, C-6a), 124.4 (d, C-5), 154.0 (s, C-3a), 162.3 (s, $\mathrm{CO}_{2}$ ), 170.7 (s, C-2). Anal. Calcd. for $\mathrm{C}_{13} \mathrm{H}_{9} \mathrm{NO}_{2} \mathrm{~S}_{3}$ (307.42): C, 49.23; H, 4.51; N, 10.44. Found: C, 49.30; H, 4.31; N, 10.36 .

Methyl 2-[(3-hydroxypropyl)amino]thieno[2,3-d]thiazole-6-carboxylate (11). A mixture of 4 (0.30 g, $1.28 \mathrm{mmol}$ ), 3-aminopropanol (0.12 g, $1.54 \mathrm{mmol})$ and $\mathrm{K}_{2} \mathrm{CO}_{3}(0.35 \mathrm{~g}, 2.57 \mathrm{mmol})$ was heated in dry dioxane $(10 \mathrm{~mL})$ at $100{ }^{\circ} \mathrm{C}$ for $4 \mathrm{~h}$. Upon addition of dry DMSO $(8 \mathrm{~mL})$ the solution was stirred at $110^{\circ} \mathrm{C}$ for $2 \mathrm{~h}$. The solution was poured into water $(100 \mathrm{~mL})$, and the 
mixture was extracted with EtOAc. The combined organic layers were washed twice with water, and the combined aqueous layers were extracted with EtOAc. The combined organic layers were dried over $\mathrm{Na}_{2} \mathrm{SO}_{4}$, some silica gel was added, and the solvent was removed in vacuo. The crude product was purified by column chromatography (silica gel/product 30:1, PE/EtOAc 5:1) to give brown crystals $11(0.09 \mathrm{~g}, 25 \%)$; mp $118-120{ }^{\circ} \mathrm{C}\left(\right.$ EtOAc); ${ }^{1} \mathrm{H}$ NMR (DMSO- $\left.d_{6}\right): \delta 1.76$ (quint., $2 \mathrm{H}, \mathrm{CH}_{2} \mathrm{CH}_{2} \mathrm{CH}_{2}, 3 \mathrm{~J}=7 \mathrm{~Hz}$ ), 3.38 (broad q, $2 \mathrm{H}, \mathrm{NCH}_{2},{ }_{3} \mathrm{~J}=7 \mathrm{~Hz}$ ), $3.50\left(\mathrm{t}, 2 \mathrm{H}, \mathrm{CH}_{2} \mathrm{OH}\right.$ ), $3.83(\mathrm{~s}$, $\left.3 \mathrm{H}, \mathrm{OCH}_{3}\right), 4.50$ (broad s, $\left.1 \mathrm{H}, \mathrm{OH}\right), 7.90(\mathrm{~s}, 1 \mathrm{H}, \mathrm{H}-5), 8.07(\mathrm{t}, 1 \mathrm{H}, \mathrm{NH}, 3 \mathrm{~J}=5 \mathrm{~Hz}) ;{ }^{13} \mathrm{C} \mathrm{NMR}$ (DMSO-d $\left.)_{6}\right): \delta 31.8\left(\mathrm{t}, \mathrm{CH}_{2} \mathrm{CH}_{2} \mathrm{CH}_{2}\right), 41.4\left(\mathrm{t}, \mathrm{NCH}_{2}\right), 52.1\left(\mathrm{q}, \mathrm{OCH}_{3}\right), 58.2\left(\mathrm{t}, \mathrm{CH}_{2} \mathrm{OH}\right), 120.0(\mathrm{~s}$, C-6), 123.5 (s, C-6a*), 125.3 (d, C-5*), 152.8 (s, C-3a), 161.6 (s, $\mathrm{CO}_{2}$ ), 172.3 (s, C-2). Anal. Calcd. for $\mathrm{C}_{10} \mathrm{H}_{12} \mathrm{~N}_{2} \mathrm{O}_{3} \mathrm{~S}_{2}$ (272.35): C, 44.10; H, 4.44; N, 10.29. Found: C, 44.12; H, 4.35; N, 10.09 .

Methyl 2-[bis(2-hydroxyethyl)amino]thieno[2,3-d]thiazole-6-carboxylate (12). A solution of $4(0.50 \mathrm{~g}, 2.13 \mathrm{mmol})$ in dry DMF $(10 \mathrm{~mL})$ was heated to $100{ }^{\circ} \mathrm{C}$. Diethanolamine $(0.56 \mathrm{~g}$, $5.35 \mathrm{mmol}$ ) was added dropwise, and the reaction mixture was stirred at $100{ }^{\circ} \mathrm{C}$ for $7 \mathrm{~h}$ and subsequently at room temperature for $16 \mathrm{~h}$. The solution was poured into water $(500 \mathrm{~mL})$, and the mixture was extracted with EtOAc. The combined organic layers were washed with water, dried over $\mathrm{Na}_{2} \mathrm{SO}_{4}$, some silica gel was added, and the suspension was evaporated. The crude product was purified by column chromatography (silica gel/product 30:1, PE/EtOAc 5:1, increasing EtOAc content) to give 12 as brown crystals $(0.22 \mathrm{~g}, 34 \%) ; \mathrm{mp} 121-124{ }^{\circ} \mathrm{C}$ (EtOAc); ${ }^{1} \mathrm{H}$ NMR (DMSO-d $\left.)_{6}\right): \delta 3.50-3.75\left[\mathrm{~m}, 8 \mathrm{H}, \mathrm{N}\left(\mathrm{CH}_{2} \mathrm{CH}_{2} \mathrm{OH}\right)_{2}\right], 3.84\left(\mathrm{~s}, 3 \mathrm{H}, \mathrm{OCH}_{3}\right), 4.90(\mathrm{t}, 2 \mathrm{H}, 2 \times$ $\mathrm{OH}, 3 \mathrm{~J}=5.5 \mathrm{~Hz}$ ), 7.97 (s, $1 \mathrm{H}, \mathrm{H}-5) ;{ }^{13} \mathrm{C}$ NMR (DMSO-d $\left.d_{6}\right): \delta 52.2\left(\mathrm{q}, \mathrm{OCH}_{3}\right), 54.3\left(\mathrm{t}, 2 \mathrm{NCH}_{2}\right.$ ), 62.8 (t, $2 \mathrm{OCH}_{2}$ ), 120.3 (s, C-6), 123.5 (s, C-6a*), 125.6 (d, C-5*), 153.3 (s, C-3a), 161.6 (s, $\mathrm{CO}_{2}$ ), 173.1 (s, C-2). Anal. Calcd. for $\mathrm{C}_{11} \mathrm{H}_{14} \mathrm{~N}_{2} \mathrm{O}_{4} \mathrm{~S}_{2}$ (302.37): C, 43.70; H, 4.67; N, 9.26. Found: C, 43.84; H, 4.69; N, 9.04.

Methyl 2-(dimethylamino)thieno[2,3-d]thiazole-6-carboxylate (13). This compound is a byproduct in the synthesis of $\mathbf{1 2}$ and was isolated as the first fraction eluted from column chromatography (silica gel/product 30:1, PE:EtOAc 5:1, gradient elution to PE/EtOAc 1:1): yellow crystals $13(0.08 \mathrm{~g}, 15 \%)$; mp 96-98 ${ }^{\circ} \mathrm{C}$ (diisopropyl ether); ${ }^{1} \mathrm{H}$ NMR $\left(\mathrm{CDCl}_{3}\right): \delta 3.12[\mathrm{~s}$,

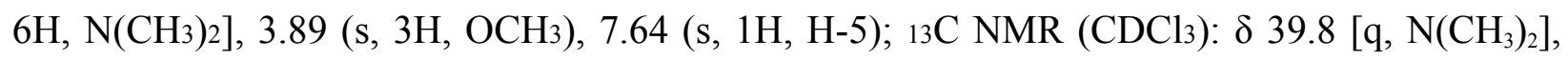
$51.8\left(\mathrm{q}, \mathrm{OCH}_{3}\right), 121.7$ (s, C-6*), 124.0 (s, C-6a*), 124.7 (d, C-5), 153.7 (s, C-3a), $162.2\left(\mathrm{~s}, \mathrm{CO}_{2}\right)$, 174.2 (s, C-2). Anal. Calcd. for $\mathrm{C}_{9} \mathrm{H}_{10} \mathrm{~N}_{2} \mathrm{O}_{2} \mathrm{~S}_{2}$ (242.32): C, 44.61; H, 4.16; N, 11.56. Found: C, 44.67; H, 3.98; N, 11.34 .

Methyl 2-[[3-(dimethylamino)propyl]amino]thieno[2,3- $d$ ] thiazole-6-carboxylate (14). Methyl 2-chlorothieno[2,3-d] thiazole-6-carboxylate 4 (2.00 g, 8.56 mmol), 3-(dimethylamino)propylamine $(1.31 \mathrm{~g}, 12.82 \mathrm{mmol})$ and $\mathrm{K}_{2} \mathrm{CO}_{3}(2.37 \mathrm{~g}, 17.15 \mathrm{mmol})$ were heated in dry DMSO $(25 \mathrm{~mL})$ at $100{ }^{\circ} \mathrm{C}$ for $1 \mathrm{~h}$. The reaction mixture was poured into water $(500 \mathrm{~mL})$ and extracted with EtOAc. The combined organic layers were washed with water, dried over $\mathrm{Na}_{2} \mathrm{SO}_{4}$, and the solvent was removed in vacuo. The product was purified by flash column chromatography (silica gel/product 30:1, methanol) to give $\mathbf{1 4}$ as brown crystals (1.20 g, 47\%); mp 91-94 ${ }^{\circ} \mathrm{C}$ (EtOAc); ${ }^{1} \mathrm{H}$ NMR (DMSO- $d 6$ ): $\delta 1.73$ (quint., $2 \mathrm{H}, \mathrm{CH}_{2} \mathrm{CH}_{2} \mathrm{CH}_{2}, 3 \mathrm{~J}=6 \mathrm{~Hz}$ ), 2.16 
$\left(\mathrm{s}, 6 \mathrm{H}, \mathrm{N}\left(\mathrm{CH}_{3}\right)_{2}\right), 2.28\left(\mathrm{t}, 2 \mathrm{H}, \mathrm{CH}_{2} \mathrm{~N}\left(\mathrm{CH}_{3}\right)_{2}, 3 \mathrm{~J}=6 \mathrm{~Hz}\right), 3.31$ (q, 2H, $\left.\mathrm{NHCH}_{2}, 3 \mathrm{~J}=6 \mathrm{~Hz}\right), 3.84$ (s, $\left.3 \mathrm{H}, \mathrm{OCH}_{3}\right), 7.90(\mathrm{~s}, 1 \mathrm{H}, \mathrm{H}-5), 8.10(\mathrm{t}, 1 \mathrm{H}, \mathrm{NH}, 3 \mathrm{~J}=3 \mathrm{~Hz}) ;{ }^{13} \mathrm{C} \mathrm{NMR}\left(\mathrm{DMSO}-d_{6}\right): \delta 26.5(\mathrm{t}$, $\left.\mathrm{CH}_{2} \mathrm{CH}_{2} \mathrm{CH}_{2}\right), 42.5$ (t, $\left.\mathrm{CH}_{2} \mathrm{~N}\left(\mathrm{CH}_{3}\right)_{2}\right), 45.1$ [q, N( $\left.\mathrm{CH}_{3}\right)_{2}$ ], 52.0 (q, $\left.\mathrm{OCH}_{3}\right), 56.4$ (t, $\left.\mathrm{NHCH}_{2}\right), 120.0$ (s, C-6), 123.5 (s, C-6a*), 125.3 (d, C-5*), 152.8 (s, C-3a), 161.6 (s, CO2), 172.2 (s, C-2). Anal. Calcd. for $\mathrm{C}_{12} \mathrm{H}_{17} \mathrm{~N}_{3} \mathrm{O}_{2} \mathrm{~S}_{2}$ (299.42): C, 48.14; H, 5.72; N, 14.03. Found: C 47.93, H 5.48, N 13.76.

Methyl 2-methoxythieno[2,3-d]thiazole-6-carboxylate (15). Methyl 2-chlorothieno-[2,3d] thiazole-6-carboxylate $4(0.10 \mathrm{~g}, 0.43 \mathrm{mmol})$ was slowly added to a solution obtained from sodium $(0.02 \mathrm{~g}, 0.64 \mathrm{mmol})$ and dry $\mathrm{MeOH}(10 \mathrm{~mL})$. After stirring at $50{ }^{\circ} \mathrm{C}$ for $4 \mathrm{~h}$ and thereafter at room temperature overnight, the reaction mixture was evaporated to dryness. The residue was dissolved in water and extracted with $\mathrm{CH}_{2} \mathrm{Cl}_{2}$. The organic layer was dried over $\mathrm{Na}_{2} \mathrm{SO}_{4}$, and the solvent was removed in vacuo. The product was purified by column chromatography (silica gel/product 30:1, PE/EtOAc 10:1) to give 15 as yellow crystals $(0.04 \mathrm{~g}$, 40\%); mp 78-80 ${ }^{\circ} \mathrm{C} ;{ }^{1} \mathrm{H} \mathrm{NMR}\left(\mathrm{CDCl}_{3}\right): \delta 3.93\left(\mathrm{~s}, 3 \mathrm{H}, \mathrm{CO}_{2} \mathrm{CH}_{3}\right), 4.16\left(\mathrm{~s}, 3 \mathrm{H}, \mathrm{OCH}_{3}\right) 7.92(\mathrm{~s}, 1 \mathrm{H}$, $\mathrm{H}-5) ;{ }^{13} \mathrm{C} \mathrm{NMR}\left(\mathrm{CDCl}_{3}\right): \delta 52.2\left(\mathrm{q}, \mathrm{CO}_{2} \mathrm{CH}_{3}\right), 58.9$ (q, $\left.\mathrm{OCH}_{3}\right), 124.5$ (s, C-6), $128.0(\mathrm{C}-5, \mathrm{C}-6 \mathrm{a})$, 148.6 (s, C-3a), 161.9 (s, $\left.\mathrm{CO}_{2}\right), 177.9$ (s, C-2).

\section{Acknowledgements}

Financial support for this project by Novartis Crop Protection is highly appreciated.

\section{References}

1. For reviews see: (a) Kuc, J. Bioscience 1982, 32, 854. (b) Kuc, J. In Ed. Active Defense Mechanisms in Plants, Wood, R. K. S., Plenum Press: New York, 1982; p. 157. (c) Kuc, J. In The Dynamics of Host Defense, Bailey, J. A.; Everall, G.J. Eds. Academic Press: Sydney, 1983, p 191. (d) Kuc, J. In Chet, J. Ed. Innovative Approaches to Plant Disease Control, Wiley: New York, 1987, p 255. (e) Metraux, J. P.; Ahl Goy, P.; Staub, T.; Speich, J.; Steinemann, A.; Ryals, J.; Ward, E. Advances in Molecular Genetics of Plant-Microbe Interactions 1991, 1, 432. (f) Kessmann, H.; Staub, T.; Ligon, J.; Oostendorp, M.; Ryals, J. European J. Plant Pathology 1994, 100, 359. (g) Kessmann, H.; Staub, T.; Hofmann, C.; Maetzke, T.; Herzog, J. Annu. Rev. Phytopathol. 1994, 32, 439. (h) Sisler, H. D.; Ragsdale, N. N. In H. Lyr Ed. Modern Selective Fungicides, Gustav Fischer, Verlag: Jena, 1995; p 543.

2. (a) Stanetty, P.; Kunz, W. EP 780, 394 A1, 1997; Chem. Abstr. 1997, 127 121735. (b) Stanetty, P.; Kremslehner, M.; Voellenkle, H. J. Chem. Soc. Perkin Trans. I, 1998, 853; (c) Stanetty, P.; Mihovilovic, M. D. Monatsh. Chem. 1999, 130, 573.

3. Stanetty, P.; Görner, E.; Mihovilovic, M. D. J. Heterocycl. Chem. 1999, 36, 761.

4. Kozikowski, A. P.; Tückmantel, W. Helv. Chim. Acta. 1994, 77, 1256.

5. Cho, Y. H.; Park, J. C. Tetrahedron Lett. 1997, 38, 8331. 